\title{
Prevención de la infección por Salmonella enterica subespecie enterica serotipo Enteritidis (Salmonella Enteritidis) en pollos mediante un bacteriófago ${ }^{\#}$
}

\author{
Prevention of Salmonella enterica subspecie enterica serotype Enteritidis \\ (Salmonella Enteritidis) infection in chickens using a bacteriophage

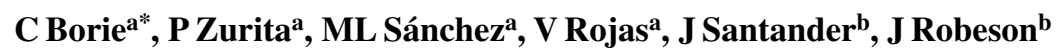 \\ ${ }^{a}$ Facultad de Ciencias Veterinarias y Pecuarias, Universidad de Chile, Santiago, Chile. \\ bInstituto de Biología, Pontificia Universidad Católica de Valparaíso, Valparaíso, Chile.
}

\section{SUMMARY}

\begin{abstract}
Infections caused by Salmonella enterica subspecies enterica serotype Enteritidis (Salmonella Enteritidis) are an important cause of foodborne diseases, epidemiologically associated with the consumption of poultry products. Since antibiotic treatments cause the appearance of multiresistant strains, phages can be used as an alternative method for controlling $S$. Enteritidis in the poultry industry. The aim of this study was to evaluate the effect of the bacteriophage $\mathrm{f} 3 \alpha \mathrm{SE}$ on the incidence of Salmonella Enteritidis in chickens. 15 broiler chickens of 10 days of age were arranged into 5 groups. Groups A and B received $1 \mathrm{ml}$ of a phage suspension orally containing $10^{6}$ and $10^{7} \mathrm{PFU} /$ dose, respectively. Two hours later, the birds were challenged orally with $1 \mathrm{ml}$ of Salmonella Enteritidis ( 4 x $10^{6} \mathrm{CFU} /$ dose). The control group (C) only received the phage (10 $\left.{ }^{7} \mathrm{PFU} / \mathrm{dose}\right)$ and the control group D was infected with Salmonella Enteritidis ( 4 x $\left.10^{6} \mathrm{CFU} / \mathrm{dose}\right)$; group E remained untreated and constituted the healthy control. Ten days post challenge, the chickens were euthanised by $\mathrm{CO}_{2}$ inhalation and samples of intestine and organs were obtained for the re-isolation of the challenge strain and phage. The incidence of infection by Salmonella Enteritidis decreased $(\mathrm{P}=0.028)$ in the group that received $10^{7} \mathrm{PFU} / \mathrm{dose}(7 / 15$ chickens) unlike the group that received a $10^{6} \mathrm{PFU}$ dose ( $8 / 15$ chickens). The decrease in the incidence of Salmonella Enteritidis in chickens by using the phage f3 $\alpha \mathrm{SE}$, indicates that it is possible to consider such phages as useful agents in the control of Salmonella Enteritidis infections.
\end{abstract}

Palabras clave: Salmonella, pollos, bacteriófagos.

Key words: Salmonella, chickens, bacteriophage.

\section{INTRODUCCION}

Salmonella enterica subespecie enterica serotipo Enteritidis (SE) es un enteropatógeno frecuentemente involucrado en brotes de infecciones transmitidas por alimentos, asociadas al consumo de alimentos de origen aviar, tales como huevos y carnes insuficientemente cocidas (Prado y col 2002, Molback y Neimann 2002). En el sector avícola, la infección puede provocar serios problemas tanto en las gallinas de postura como en broiler; la infección a temprana edad puede producir mortalidad y disminución de la eficiencia productiva en las aves adultas, no obstante, la mayoría de las aves quedan en estado de portadoras asintomáticas (Barrow 1991, Gast 1994). Entre las medidas tradicionales de control y prevención destacan: utilización de prebióticos y probióticos, medidas de bioseguridad (control de roedores y aves migratorias), vacunación y antimicrobianos. Ninguna de estas medidas, aisladas o en asociación, ha logrado la eficiencia esperada por el sector avícola. Actualmente, el escenario de la salmonelosis se ha

Aceptado: 31.10.2007.

\# Financiado por Proyecto FIV 3718.

* Casilla 2 Correo 15, La Granja, Santiago, Chile; cborie@uchile.cl complicado con la emergencia de cepas multirresistentes a antimicrobianos, limitando con ello el abordaje profiláctico en animales. Frente a este complejo escenario, reaparece el antiguo concepto de utilizar bacteriófagos líticos como terapia clínica y como medida preventiva.

Los bacteriófagos líticos, descubiertos por Twort en 1915 y D'Herelle en 1917, son virus que replican al interior de una bacteria y la lisan. Cada tipo de fago reconoce una bacteria específica, se une a receptores de superficie, inyecta su genoma al interior de la célula para luego producir cientos de partículas virales que, gracias a sus enzimas, logran romper la pared celular bacteriana y liberar su progenie. En este proceso la célula bacteriana muere y el número de fagos se incrementa, amplificando el efecto antibacteriano (Gòrski y Weber-Dabrowska 2005, Hudson y col 2005). Hasta la fecha no se ha informado que los fagos produzcan lisis en células animales, humanas ni en plantas (Sulakvelidze y col 2001, Dabrowska y col 2005). Los primeros trabajos en animales se realizaron en la década del 80 en Gran Bretaña, con ratas experimentalmente inoculadas con Escherichia coli y, posteriormente, en ovejas, cerdos y terneros con diarrea por E. coli, logrando disminuir el número de patógenos en el tracto digestivo (Sulakvelidze y col 2001). Posteriormente, aparecen estudios en pollos y peces, con resultados promisorios en 
el área de la terapia médica (Nakai y col 1999, Park y col 2000 , Huff y col 2003, Fiorentin y col 2005, Toro y col 2005, Wagenaar y col 2005).

La industria de alimentos también ha utilizado con éxito fagos para la reducción de enteropatógenos importantes en salud pública, específicamente en queso Cheddar (Modi y col 2001), manzanas y melones (Lerverentz y col 2001), canales de pollos broiler (Higgins y col 2005) y piel de pollos (Goode y col 2003). En agosto del año 2006, Estados Unidos de Norteamérica aprobó la utilización de una mezcla de 7 bacteriófagos líticos, contra Listeria monocytogenes, como aditivo alimenticio (Bren 2007).

En infecciones por Salmonella spp en aves, Sklar y Joerger (2001), Toro y col (2005) y Fiorentin y col (2005) demuestran que la administración oral de una mezcla de bacteriófagos permite la reducción de la colonización a nivel intestinal en pollos broiler experimentalmente infectados, disminuyendo con ello la posibilidad de contaminación en los productos derivados de ellos. La utilización de fagos líticos presenta numerosas ventajas, entre las cuales destacan la facilidad y bajo costo de su aislamiento y preparación, su escasa a nula acción lítica sobre bacterias de flora normal, su facilidad de administración por vía oral, respiratoria, intramuscular e intraperitoneal y menor tasa de resistencia bacteriana que los antimicrobianos (Sklar y Joerger 2001, Huff y col 2003, Górski y Weber-Dabrowska 2005, Toro y col 2005).

En Chile, Santander y Robeson (2002) aislaron fagos líticos contra Salmonella Enteritidis a partir de aguas servidas de planteles avícolas de la V Región. Uno de los fagos aislados, denominado $\mathrm{f} 3 \alpha \mathrm{SE}$, es altamente lítico in vitro frente a cepas de Salmonella Pullorum y Salmonella Enteritidis, logrando disminuir el recuento bacteriano de $10^{8}$ unidades formadoras de colonias $/ \mathrm{ml}(\mathrm{UFC} / \mathrm{ml})$ a $10^{3}$ UFC/ml en 100 minutos. También, f $3 \alpha$ SE demostró ser lítico en cepas de Salmonella Pullorum, de una manera similar que Salmonella Enteritidis. Este fago tolera un amplio rango de condiciones fisicoquímicas como congelamiento $-20{ }^{\circ} \mathrm{C}$ y cambios de temperaturas entre 4 y $50{ }^{\circ} \mathrm{C}, \mathrm{pH}$ entre $4-12$, alta estabilidad en agua y condiciones de almacenamiento, que indican que posee potencial para ser utilizado en la industria avícola (Santander y Robeson 2007). Por otra parte, estudios in vivo utilizando el modelo animal Caenorhabiditis elegans demostraron que el fago $\mathrm{f} 3 \alpha \mathrm{SE}$ fue eficaz en tratamientos de fagoprofilaxis contra Salmonella Enteritidis y Salmonella Pullorum (Santander y Robeson 2004). El objetivo de este trabajo fue determinar la capacidad del fago $\mathrm{f} 3 \alpha \mathrm{SE}$, para reducir la incidencia de Salmonella Enteritidis en pollos experimentalmente infectados.

\section{MATERIAL Y METODOS}

\section{CEPA DESAFIO}

Se utilizó una cepa de Salmonella Enteritidis aislada de una gallina y donada por el Servicio Agrícola y Ganadero
(SAG) de Chile. Se seleccionó una doble mutante espontánea resistente a ácido nalidíxico $\left(\mathrm{Nal}^{\mathrm{r}}\right)$ y rifampicina $\left(\right.$ Rif $\left.^{\mathrm{r}}\right)$ y se incrementó su virulencia mediante seis pasajes en pollos. Se utilizó una dosis mínima infectante de 4 x $10^{6} \mathrm{UFC} / \mathrm{ml}$.

\section{BACTERIOFAGO}

El fago f $3 \alpha$ SE fue aislado y caracterizado por Santander y Robeson (2002) como un virus morfotipo 1. Para la dosificación en las aves, el fago se suspendió en agua destilada hasta obtener concentraciones de $10^{6}$ unidades formadoras de placas $/ \mathrm{ml}$ (UFP/ml) y $10^{7} \mathrm{UFP} / \mathrm{ml}$, correspondientes a una multiplicidad de infección (MOI) de 1 y 10 , respectivamente.

\section{ANIMALES}

Se trabajó con pollos broiler de un día de edad, adquiridos en un plantel de la Región Metropolitana y mantenidos con agua y alimento no medicado ad libitum en una Unidad para Animales de Experimentación. Los animales se criaron y manejaron de acuerdo a recomendaciones del Comité de Bioética de la Facultad de Ciencias Veterinarias y Pecuarias, Universidad de Chile. Todas las aves fueron negativas a Salmonella spp mediante cultivo tradicional de deposiciones obtenidas al día 3 de edad y fueron mantenidas bajo estrictas condiciones de bioseguridad para evitar una posible contaminación con cepas de campo.

\section{DISEÑO EXPERIMENTAL}

A los 10 días de edad, los pollos fueron separados en 5 grupos de 15 animales cada uno (cuadro 1). El grupo A recibió por vía oral $1 \mathrm{ml}$ del bacteriófago $\mathrm{f} 3 \alpha \mathrm{SE}$ en una dosis de $10^{6} \mathrm{UFP} / \mathrm{ml}$ (MOI 1) y los grupos B y $\mathrm{C}$ recibieron $10^{7} \mathrm{UFP} / \mathrm{ml}$ (MOI 10). Dos horas después, las aves de

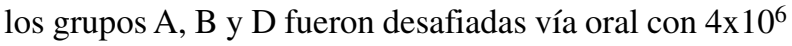

Cuadro 1. Diseño experimental. Experimental design.

\begin{tabular}{|c|c|c|}
\hline Grupo & $\begin{array}{l}\text { Número } \\
\text { de aves }\end{array}$ & Tratamiento \\
\hline A & 15 & $\begin{array}{l}\text { Tratados con } 10^{6} \text { UFP de } \mathrm{BF}^{*} \text { e infectados } \\
\text { con } \mathrm{SE}^{*}\end{array}$ \\
\hline $\mathrm{B}$ & 15 & $\begin{array}{l}\text { Tratados con } 10^{7} \text { UFP de BF e infectados } \\
\text { con SE }\end{array}$ \\
\hline $\mathrm{C}$ & 15 & $\begin{array}{l}\text { Tratados con } 10^{7} \text { UFP de BF y No } \\
\text { infectados con SE }\end{array}$ \\
\hline $\mathrm{D}$ & 15 & No tratados con BF e infectados con SE \\
\hline $\mathrm{E}$ & 15 & No tratados con BF ni infectados con SE \\
\hline
\end{tabular}


$\mathrm{UFC} / \mathrm{ml}$ de Salmonella Enteritidis Nal ${ }^{\mathrm{r}} \mathrm{Rif}^{\mathrm{r}}$. Las aves del grupo D sólo recibieron el desafío con SE. El grupo E no recibió tratamiento con fago ni desafío bacteriano y se utilizó para detectar una posible transmisión accidental de fago y/o bacteria entre las unidades experimentales. La administración por vía oral, tanto de fagos como de la cepa desafío, se realizó mediante una cánula semirrígida adosada a una jeringa; para evitar reflujo, las aves se mantuvieron en ayuno de 4 horas previo a la inoculación.

A los 10 días postinfección (20 días de edad), todos los pollos fueron eutanasiados por inhalación de $\mathrm{CO}_{2}$ (Beaver 2001) y de ellos se obtuvieron muestras individuales tanto de intestino completo como de "pool" de órganos (bazo, hígado y corazón). Las muestras se procesaron para detección de fagos y cepa desafío. La bacteriología cualitativa se realizó de acuerdo a la metodología descrita por Murray y Barton (1993), utilizando como enriquecimiento caldo Rappaport-Vassiliadis (Difco) y como medio selectivo, agar XLD (Difco) adicionado de ácido nalidíxico (Laboratorio Chile, $20 \mu \mathrm{g} / \mathrm{ml}$ ) y rifampicina (Laboratorio Chile, $20 \mu \mathrm{g} / \mathrm{ml}$ ). Todas las colonias sospechosas (centro negro y borde transparente) se identificaron mediante el kit de diagnóstico rápido BBL Crystal (Becton, Dickinson and Company) y por aglutinación con antisuero grupo D1 (Difco, St. Louis MO).

El aislamiento del fago a partir de las muestras sembradas en caldo Rappaport-Vassiliadis se realizó de acuerdo a lo descrito por Santander y Robeson (2002). Un ml de cada muestra se agregó a un caldo Luria Bertani (Difco) adicionado de rifampicina $(100 \mu \mathrm{g} / \mathrm{ml})$ y de $0,5 \mathrm{ml}$ de una suspensión de SE ATCC 13076 Rif $^{\mathrm{T}}$ de 24 horas de incubación a $37^{\circ} \mathrm{C}$. Luego de una agitación durante toda la noche, $1 \mathrm{ml}$ se centrifugó a 10.000 x $g$ durante 5 minutos. El sobrenadante se trató con cloroformo y fue sembrado sobre un cultivo de SE ATCC 13076, utilizando el método de la doble capa de agar; luego de una incubación a $37{ }^{\circ} \mathrm{C}$ se observó la formación de placas líticas.

\section{ANALISIS DE RESULTADOS}

La incidencia de infección por Salmonella Enteritidis se determinó mediante los resultados de los cultivos de intestino y/o "pool" de órganos de las aves infectadas. Se analizaron las diferencias de proporciones entre los grupos tratados y el grupo control de infección, mediante $\mathrm{X}^{2}$ utilizando el programa estadístico Infostat version 2004 (Grupo Infostat, FCA, Universidad Nacional de Córdoba, Argentina).

\section{RESULTADOS Y DISCUSION}

Independiente de la muestra analizada, en las aves del grupo control de infección (grupo D), inoculadas con la dosis $4 \times 10^{6} \mathrm{UFC} / \mathrm{ml}$ de SE, se observó un alto porcentaje de infección (86,6\%). La infección no alcanzó al 100\% de las aves, probablemente debido a la baja virulencia de



Grupo A: tratado con $10^{6} \mathrm{UFP} / \mathrm{ml}$ del bacteriófago (MOI 1). Grupo B: tratado con $10^{7} \mathrm{UFP} / \mathrm{ml}$ del bacteriófago (MOI 10). Grupo D: solo infectado con SE. MOI*: Multiplicidad de infección

Figura 1. Aislamiento de Salmonella Enteritidis (SE) de pollos experimentalmente tratados con un bacteriófago e infectados con $\mathrm{SE}$, según tipo de muestra, 10 días posterior a la infección.

Salmonella Enteritidis (SE) isolation from experimentally bacteriophage treated and SE infected chickens, according to type of samples, 10 days after infection.

la cepa y no a la dosis utilizada ya que ésta se encuentra dentro del rango más alto señalado en estudios similares. En este grupo se aisló la cepa desafío en el 66,6\% de las muestras de "pool" de órganos y en $86,6 \%$ de las muestras de intestino completo (figura 1).

El efecto del tratamiento con el fago f $3 \alpha \mathrm{SE}$ (MOI 1 y 10) sobre la incidencia de la infección se observa en la figura 1. En el grupo A, que recibió el fago con una MOI de 1 ( $10^{6} \mathrm{UFP} /$ dosis), se infectaron 8 de 15 pollos $(53,3 \%$, $\mathrm{p}=0,0619)$ y 13 de 15 en el grupo control de infección $(86,6 \%)$. El aislamiento de la cepa desafío en el grupo A fue más frecuente en las muestras de intestino completo que en "pool" de órganos (figura 1). En el caso del grupo $\mathrm{B}$, que recibió el fago con una MOI de $10\left(10^{7} \mathrm{UFP} /\right.$ dosis), se infectaron 7 de 15 aves $(46,6 \%, p=0,028)$. El aislamiento de SE en intestino y "pool" de órganos de las aves del grupo B fue similar en ambos tipos de muestras (figura 1).

Diversas experiencias han demostrado que la multiplicidad de infección (MOI) juega un rol importante en el éxito de las fagoterapias. Goode y col (2003) utilizaron MOI en rangos de $10^{2}$ a $10^{5}$ para reducir la cantidad de Salmonella spp en piel de pollos, pero sólo la MOI $10^{5}$ logró eliminar la bacteria completamente de la piel de las aves. De la misma forma, Huff y col (2006), utilizando multiplicidades de $10^{2}, 10^{4}, 10^{6}$ y $10^{8}$ UFP del fago SPRO2, concluyeron que la MOI de $10^{4}$ fue capaz de reducir significativamente la tasa de mortalidad de pollos infectados con una cepa de E. coli. Aunque los fagos son capaces 
de multiplicarse en la célula hospedadora, las terapias con bacteriófagos de mayor éxito han sido aquellas que utilizan una MOI elevada (Goode y col 2003, Huff y col 2003 , Fiorentin y col 2005, Wagenaar y col 2005). Hasta la fecha, no existen en la literatura evidencias que sugieran utilizar dosis bajas de fagos. La menor actividad lítica del fago sobre Salmonella a nivel intestinal, en las aves que recibieron una MOI de 1, se podría explicar, en parte, por la presencia en la mucosa intestinal de un medio viscoso que actuaría como barrera física, impidiendo el encuentro entre bacteria y fago (Sklar y Joerger 2001).

El objetivo de este trabajo fue prevenir la colonización intestinal de Salmonella y por ello las aves recibieron el fago antes y no después de la infección con SE. En experiencias con otras bacterias, Huff y col (2003), empleando E. coli, observaron una disminución significativa de la mortalidad en pollos cuando administraron el fago, mediante aerosoles, inmediatamente después de la infección; cuando los desafíos con E. coli fueron 24-48 horas después no se observaron buenos resultados. Sin embargo, cuando la administración del fago fue por vía intramuscular, lograron buenos resultados con el desafío realizado 24-48 horas después. Por otro lado, experiencias realizadas en pollos para prevenir la colonización intestinal de Campylobacter jejuni, donde el fago se dosificó tan temprano como a los 3 días antes del desafío, sólo se logró retardar la colonización pero no prevenirla (Wagenaar y col 2005). Barrow y col (1998) señalan que fagos dosificados en pollos hasta 2 días antes de la inoculación con E. coli otorgaron protección, pero no cuando se dosificaron cinco días antes del desafío.

En relación a la detección del virus, en los grupos A, B y $\mathrm{C}$ se aislaron tanto en intestino como en órganos (cuadro 2), demostrando que este fago presenta una permanencia sistémica de al menos 10 días, constituyendo esto una potencial ventaja en la prevención de futuras reinfecciones en las aves.

En el grupo E, control sano, las muestras fueron negativas a Salmonella Enteritidis y a bacteriófago, demostrando ausencia de contaminación accidental entre los diferentes grupos experimentales.

Durante los 10 días de la experiencia los animales no evidenciaron cambios de comportamiento ni manifestaciones clínicas. En la necropsia no se observaron lesiones macroscópicas sugerentes de alguna patología, lo que refuerza el concepto de inocuidad de los fagos.

Cuadro 2. Detección de fagos en pollos tratados con un bacteriófago e infectados con Salmonella Enteritidis, 10 días posterior a la infección.

Phage detection in bacteriophage treated and infected chickens with Salmonella Enteritidis, 10 days after infection.

\begin{tabular}{ccc}
\hline Grupo & Detección en órganos & Detección en intestino \\
\hline $\mathrm{A}$ & $3 / 15$ & $8 / 15$ \\
$\mathrm{~B}$ & $9 / 15$ & $3 / 15$ \\
$\mathrm{C}$ & $6 / 15$ & $6 / 15$ \\
\hline
\end{tabular}

Los resultados obtenidos, sumados a la facilidad de aislamiento y bajo costo de preparación del fago, su termotolerancia y estabilidad en agua destilada, permiten sugerir que los fagos podrían ser considerados como una herramienta alternativa, complementaria a los métodos de control y prevención de Salmonella actualmente vigentes.

\section{RESUMEN}

Las infecciones por Salmonella Enteritidis son una causa importante de enfermedades transmitidas por alimentos, epidemiológicamente asociada al consumo de productos derivados de las aves. Ya que el tratamiento con antimicrobianos causa la aparición de cepas multirresistentes, los fagos pueden ser usados como método alternativo para controlar $S$. Enteritidis en la industria avícola. El propósito de este trabajo fue evaluar el efecto del bacteriófago f $3 \alpha \mathrm{SE}$ sobre la incidencia de $S$. Enteritidis en pollos. Se formaron 5 grupos de 15 pollos broiler de 10 días de edad; los grupos experimentales A y B recibieron vía oral $1 \mathrm{ml}$ del fago, conteniendo $10^{6}$ y $10^{7} \mathrm{UFP} /$ dosis, respectivamente. Dos horas después, las aves fueron desafiadas con $1 \mathrm{ml}$ de $S$. Enteritidis (4 x $10^{6} \mathrm{UFC} /$ dosis). El grupo C sólo recibió fagos $\left(10^{7} \mathrm{UFP} /\right.$ dosis $)$ y el grupo D sólo $S$. Enteritidis (4 x $10^{6} \mathrm{UFC} /$ dosis); el grupo E no recibió tratamiento y constituyó el grupo control sano. Diez días postinfección, los pollos recibieron eutanasia por inhalación de gas, obteniéndose por cada ave muestras de intestino y órganos para el reaislamiento de la cepa desafío y del fago. La incidencia de infección por $S$. Enteritidis se redujo en el grupo que recibió $10^{7} \mathrm{UFP} /$ dosis $(\mathrm{p}=0,028)(7 / 15$ aves $)$ del bacteriófago, no así en el grupo que recibió la dosis de $10^{6} \mathrm{UFP}$ ( $\left.\mathrm{p}=0,061\right)(8 / 15$ aves). La disminución de la incidencia de $S$. Enteritidis en pollos, lograda mediante el fago $f 3 \alpha \mathrm{SE}$, permite considerarlo como una posible alternativa al uso de antimicrobianos en el control de Salmonella.

\section{REFERENCIAS}

Barrow P. 1991. Experimental infection of chickens with Salmonella enteritidis. Avian Pathol 22, 651-669.

Barrow P, M Lovell, A Berchieri. 1998. Use of lytic bacteriophage for control of experimental Escherichia coli septicemia and meningitis chickens and calves. Clin Diagn Lab Immunol 5, 294-298.

Beaver B. 2001. 2000 Report of the American Veterinary Medical Association Panel of Euthanasia. J Am Vet Med Anim 218, 669-696.

Bren L. 2007. Bacteria-eating virus approved as food additive. FDA Consum 41, 20-22.

Dabrowska K, K Switala-Jelen, A Opolski, B Weber-Dabrowska, A Górski. 2005. Bacteriophage penetration in vertebrates. J Appl Microbiol 98, 7-13.

Fiorentin L, D Nilson, W Barioni. 2005. Oral treatment with bacteriophages reduces the concentration of Salmonella enteritidis PT4 in caecal contents of broilers. Avian Pathol 34, 258-263.

Gast R. 1994. Understanding Salmonella enteritidis in laying chickens: the contributions of experimental infection. Int J Food Microbiol 21, 107-116.

Goode D, VM Allen, PA Barrow. 2003. Reduction of experimental Salmonella and Campylobacter contamination of chicken skin by application of lytic bacteriophage. Appl Environ Microbiol 69, 5032-5036.

Gòrski A, B Weber-Dabrowska. 2005. The potential role of endogenous bacteriophages in controlling invading pathogens. Cell Mol Life Sci 62, 511-519.

Higgins JP, SE Higgins, KL Guebnther, W Huff, AM Donoghue, DJ Donoghue, BM Hargis. 2005. Use of bacteriophage treatment to reduce Salmonella in poultry products. Poultry Sci 84, 1141-1145. 
Hudson JA, C Billington, G Carey-Smith, G Greening. 2005. Bacteriophages as biocontrol agents in food. J Food Prot 68, 426-437.

Huff WE, GR Huff, NC Rath, JM Balog, AM Donoghue. 2003. Evaluation of aerosol spray and intramuscular injection of bacteriophage to treat an Escherichia coli respiratory infection. Poult Sci 82, 1108-1112.

Huff WE, GR Huff, NC Rath, AM Donoghue. 2006. Evaluation of the influence of bacteriopohage titer on the treatment of colibacillosis in broiler chicken. Poult Sci 85, 1373-1377.

Leverentz B, WS Conway, Z Alavidze, WJ Janisiewicvz, Y Fuchs, MJ Camp, E Chigladze, A Sulakvelidze. 2001. Examination of bacteriophage as a biocontrol method for Salmonella on fresh-cut fruit: a model study. J Food Prot 64, 1116-1121.

Murray CJ, M Barton. 1993. Salmonellosis bacteriology. In: Corner LA, Baugust TJ (eds). Australian standard diagnostic techniques for animal diseases. Australia, Pp 3-8.

Modi R, Y Hirvi, A Hill, MW Griffiths. 2001. Effect of phage on survival of Salmonella enteritidis during manufacture and storage of cheddar cheese made from raw and pasteurized milk. J Food Prot 64, 927-933.

Molback K, K Neimann. 2002. Risk factors for sporadic infection with Salmonella enteritidis, Denmark, 1997-1999. Am J Epidemiol $156,654-661$.

Nakai T, R Sugimoto, KH Park, S Matsuoka, K Mori, T Nishioka, K Maruyama. 1999. Protective effects of bacteriophage on experimental Lactococcus garvieae infection in Yellowtail. Dis Aquat Organ 37, 33-41.

Park SC, I Shimamura, M Fukunaga, KI Mori, T Nakai. 2000. Isolation of bacteriophages specific to a fish pathogen, Pseudomonas pleco- glossica, as a candidate for disease control. Appl Environ Microbiol 66, 1416-1422.

Prado V, V Solari, I Alvarez, C Arellano, R Vidal, M Carreño, N Madani, D Fuentes, M O'Ryan, V Muñoz. 2002. Situación epidemiológica de las enfermedades transmitidas por alimentos en Santiago de Chile: Periodo 1999-2000. Rev Med Chil 130, 495-501.

Santander J, J Robeson. 2002. Aislamiento y caracterización de bacteriófagos líticos contra Salmonella Enteritidis y su ensayo sobre Salmonella Pullorum. Acta Microbiol 8, 17-22.

Santander J, J Robeson. 2004. Phage prophylaxis against Salmonella enteritidis using Caenorhabditis elegans as an assay system. Electronic J Biotechnol 7, 11-14.

Santander J, J Robeson. 2007. Survival properties of bacteriophages active against Salmonella enterica serovars Enteritidis and Pullorum with potential for biocontrol in the poultry industry. American Society for Microbiology, April 14, Branch Meeting, Arizona-Nevada, Flagstaff, AZ, USA.

Sklar IB, RD Joerger. 2001. Attemps to utilize bacteriophage to combat Salmonella enterica serovar enteritidis infection in chickens. $J$ Food Saf 21, 15-29.

Sulakvelidze A, Z Alavidze, JG Morris. 2001. Bacteriophage therapy. Antimicrob Agents Chemother 45, 649-659.

Toro H, SB Price, S McKee, FJ Hoerr, J Khreling, M Perdue, L Bauersmeister. 2005. Use of bacteriophages in combination with competitive exclusion to reduce Salmonella from infected chickens. Avian Dis 49, 118-124.

Wagenaar JA, MAP Van Bergen, MA Mueller, TM Wassenaar, RM Carlton. 2005. Phage therapy reduces Campylobacter jejuni colonization in broilers. Vet Microbiol 109, 275-283. 
